\title{
From eye movements to actions: how batsmen hit the ball
}

\author{
Michael F. Land ${ }^{1}$ and Peter McLeod ${ }^{2}$ \\ ${ }^{1}$ School of Biological Sciences, University of Sussex, Brighton BN1 9QG, UK \\ 2 Department of Experimental Psychology, University of Oxford, South Parks Road, Oxford OX1 3UD, UK \\ Correspondence should be addressed to M.F.L. (m.f.land@sussex.ac.uk)
}

\begin{abstract}
In cricket, a batsman watches a fast bowler's ball come toward him at a high and unpredictable speed, bouncing off ground of uncertain hardness. Although he views the trajectory for little more than half a second, he can accurately judge where and when the ball will reach him. Batsmen's eye movements monitor the moment when the ball is released, make a predictive saccade to the place where they expect it to hit the ground, wait for it to bounce, and follow its trajectory for $100-200 \mathrm{~ms}$ after the bounce. We show how information provided by these fixations may allow precise prediction of the ball's timing and placement. Comparing players with different skill levels, we found that a short latency for the first saccade distinguished good from poor batsmen, and that a cricket player's eye movement strategy contributes to his skill in the game.
\end{abstract}

Looking in the right place at the right time is particularly important in ball sports such as tennis, cricket or baseball, in which a player needs to determine the future trajectory of the ball and the timing of contact with it. In most such sports, a coach's advice is to "keep your eye on the ball." However, when a ball moves fast, this strategy may not always be possible or appropriate. Here we examine the eye movements of cricket batsmen, and show that, in general, they do not watch the ball continuously. They have a distinct eye movement strategy to view the ball at crucial moments during its flight.

The rules of cricket have evolved to produce a balanced contest between the visual-motor skills of the batsman and the strength and skill of the bowler. Batting is possible (or batsmen would refuse to play), but not all the time (or bowlers would refuse to play). The abilities of the best batsmen against the fastest bowlers reveal the limits of the visual-motor system.

When a batsman plays a shot such as the pull or the hook, the bat is swung horizontally in an arc at right angles to the trajectory of the approaching ball. The batsman must judge the vertical position of the ball to within $\pm 3 \mathrm{~cm}$ (limited by the bat's width) and its time of arrival to within $\pm 3 \mathrm{~ms}$ (limited by the time the ball takes to pass the effective percussion zone of the bat $)^{1,2}$. What physical parameters determine the point and time of contact? The ball leaves the bowler's hand with forward velocity $v_{\mathrm{h} 0}$ and downward velocity $v_{\mathrm{v} 0}$ (Fig. 1). It hits the ground a distance $x_{1}$ from the batsman after time $t_{0}$, bounces up and reaches him at a height $y$ and time $t_{1}$ after the bounce. The values of $y$ and $t_{1}$ are determined by the horizontal and vertical velocities of the ball after bouncing $\left(v_{\mathrm{h} 1}\right.$ and $\left.v_{\mathrm{v} 1}\right)$ and by $x_{1}$. A fast bowler typically generates an $x_{1}$ between 10 and $0 \mathrm{~m}$, depending on the value of $v_{\mathrm{v} 0}$, the downward velocity of the ball when he releases the ball. The corresponding values of $y$ vary from 2 to $0 \mathrm{~m}$ depending on the speed of the bowler and the hardness of the ground. Here we examine the path of the ball in the vertical plane. If the batsman chooses to hit the ball, he usually 'gets in line with the ball' - that is, he moves laterally until the ball is coming toward the midline of his body, and there are straightforward visual cues available to make this judgment ${ }^{1}$. Our analysis applies to fast and medium fast bowling $(>25$ $\mathrm{m} /$ per/s). Slow bowling, where spin rather then speed is used to defeat the batsman, raises different problems ${ }^{1}$. (Definitions of cricketing terms are in Methods.)

The estimation of $y$ and $t_{1}$ from information provided by an approaching ball is a difficult problem for the batsman, because of his reaction time. It takes about $200 \mathrm{~ms}$ for even an expert batsman to adjust his shot on the basis of novel visual information $^{3}$. (In some sports, such as table tennis, reaction times may be faster ${ }^{4}$, but the inertia of the cricket bat precludes faster responses.) Therefore, his judgment must be essentially predictive, based on information available at least $200 \mathrm{~ms}$ before the ball reaches him. With a fast bowler, the ball takes about $600 \mathrm{~ms}$ to reach the batsman, so the batsman must select an appropriate trajectory for his bat based on information from the first twothirds of the ball's flight.

Views on how batsmen acquire the information they need have emphasized direct visual measurements such as image expansion (from which time to contact can be derived) and rate of change of binocular disparity ${ }^{1,5}$. However, given the speed of the ball and the batsman's reaction time, judgments using these parameters would have to be made when the ball's image and its rate of expansion were very small, which would make the possibility of obtaining millisecond accuracy from such measurements very unlikely. Also, precise determination of arrival time from image expansion requires that the object approach the eye directly, and at a constant velocity. Cricket balls change speed when they bounce, decelerate as they approach the batsman, and travel in an arc, arriving at a variety of heights but seldom level with the eye. Nevertheless, batsmen can judge the ball's arrival time to within a few milliseconds.

Here we wanted to determine what information is available to batsmen as the ball approaches them, by monitoring 


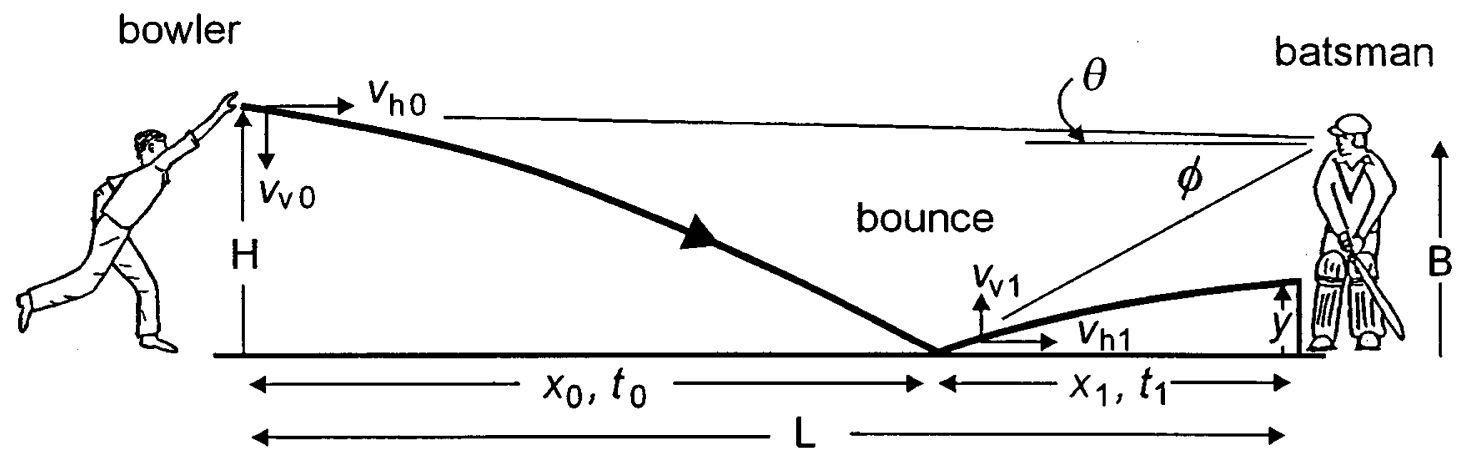

Fig. 1. The physical parameters that determine where and when the ball will reach the batsman, and the measurements he can make to determine the time and point of contact. The batsman's task is to estimate when it will reach him $\left(\mathrm{t}_{1}\right)$, and at what height (y). 0 ther definitions are in text.

their direction of their gaze. Because the best information about the position and velocity of approaching objects comes from the fovea and its immediate vicinity ${ }^{6}$, eye movements are likely to show which parts of the flight are important to the batsman. We recorded the eye movements and field of gaze during batting of three batsmen with a wide range of abilities. Our results suggest that the mechanism for estimating $y$ and $t_{1}$ is different from those previously proposed. The information required for a correctly timed hit is available from fixating on the moment of delivery and the time and place of the ball's bounce.

\section{RESULTS}

The eye movements of three batsmen were measured with a headmounted eye camera as they faced balls delivered at $25 \mathrm{~m}$ per second from a bowling machine. The camera recorded the view from the batsman's left eye, as well as the direction of the fovea's gaze (Fig. 2). The upper two-thirds of the video display gave the view ahead. Gaze direction was derived from the position of the inverted eye, which is shown in the lower third of the display (Fig. 2). Foveal gaze direction was indicated by a white dot, which has a diameter of approximately $1^{\circ}$. In frame $1(0.0 \mathrm{~s}$; Fig. 3$)$, the eye fixated on the aperture of the bowling machine (visible on frame 2 when gaze moved away from it). In frame $2(0.16 \mathrm{~s})$, the ball (black dot below gaze spot) had left the machine and fallen by about $3^{\circ}$. Frame $3(0.19 \mathrm{~s})$ was taken toward the end of the anticipatory saccade, which shifted gaze to about $10^{\circ}$ below the bowling machine, level with the future bounce point. Gaze now led the ball by about $5^{\circ}$. In frame $4(0.4 \mathrm{~s})$, at the bounce point, the gaze and the ball were within $1^{\circ}$ of each other. In frame $5(0.5 \mathrm{~s})$ gaze continued to track the ball closely, but in frame $6(0.6 \mathrm{~s})$ a saccade brought gaze to a point about $5^{\circ}$ below the ball. From frame 3 onward, the field of view moved up, indicating that the head moved down.

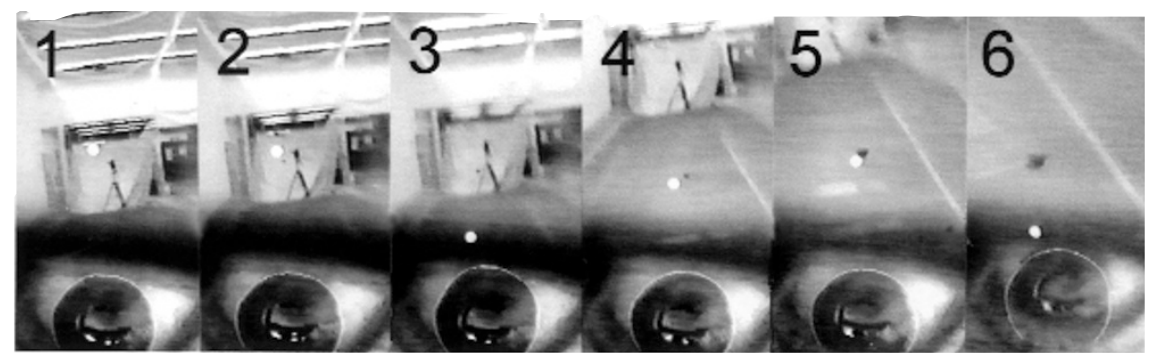

\section{Eye and head movements}

The vertical movements of the eye (relative to the head) and head (relative to external space) were recorded (Fig. 3a). The eye was almost stationary for $0.14 \mathrm{~s}$ after the ball appeared (fixating on the point from which it was delivered; Fig. 2) and then made a downward saccade of $7.5^{\circ}$. The eye then rotated upward for $0.3 \mathrm{~s}$, while the head moved downward through the same angle. At $0.5 \mathrm{~s}$, this counter-rotation stopped, and both head and eye moved rapidly down, with a large eye saccade at $0.58 \mathrm{~s}$. The bounce occurred at $0.38 \mathrm{~s}$, near the middle of the counterrotation period.

Adding the eye and head angles (Fig. 3a) gave the gaze angle, the direction of foveal regard in space (Fig. 3b, bottom, open circles). Gaze angle was compared to the location of the ball as seen from the batsman's eye (Fig. 3b, bottom, filled circles). Initially there was no gaze error (angle between ball and fovea; Fig. 3b, top), but then a small error built up before the saccade produced an error of $6^{\circ}$ in the opposite direction. Error reduced to zero as the ball bounced, and remained close to zero for another $0.2 \mathrm{~s}$ before becoming negative again. The fovea was directed to the ball at the moment of delivery, at the bounce point and for about $0.2 \mathrm{~s}$ after the bounce.

\section{Eye movement strategy}

Eyes initially focused on the point of delivery. The gaze was stationary for a period after delivery as the ball dropped into the field of view. A saccade was then made which brought the fovea below the ball, close to point where the ball would subsequently bounce. The fovea thus 'lay in wait' for the bounce. (Anticipatory saccades like these are also seen in table tennis ${ }^{7,8}$ and baseball ${ }^{9}$.) The vertical counter-rotation of eye and head (presumably driven by the vestibulo-ocular reflex) kept gaze direction roughly level for a period before and after the bounce. Then both eye and head moved rapidly down trying to track the latter part of the ball's flight. In this example, the terminal

Fig. 2. Frames from the eye-tracker video, showing the batsman's view of an approaching ball, and the direction foveal gaze as he watches it (white dot). Sequence is taken from a good-length delivery to Charlie, a competent amateur. Details of the sequence from which these frames are taken are plotted in Fig. 3. 
tracking was not very successful, and the gaze got ahead of the ball (Fig. 2, frame 6).

\section{Variation between deliveries of different lengths}

We compared the responses of three batsmen to deliveries of four different lengths (Figs. 4 and 5). The batsmen's responses were quite consistent, until they lost the ball at the end of its trajectory (small standard deviations; Fig. 4). Bounce length affected gaze movements in two ways. First, the delay before the early downward saccade increased as the ball bounced closer to the batsman. The delay increase was particularly evident in Charlie's record (Fig. 5a), where the latency increased from $0.14 \mathrm{~s}$ for the very short balls to almost $0.3 \mathrm{~s}$ for the over-pitched balls. Presumably the variable controlling this timing was the initial downward velocity of the ball on the stationary retina (Fig. 5b). Second, the amount of smooth tracking that accompanied the initial saccade increased as the length of the delivery increased. This was especially true of Mark (Fig. 4). His saccade sizes actually decreased as the ball was pitched closer to the batsman (Fig. 5a), as more of the downward movement was achieved with smooth tracking. With this combination of saccade and smooth tracking, the fovea reached the bounce point accurately and well ahead of the ball.

After the bounce, the batsmen used smooth pursuit gaze movements to track the ball. Thus, for very short balls (which bounce above the direction of gaze to the bounce point), the gaze moved upwards (Fig. 4, top), and for the over-pitched balls (whose post-bounce trajectory was below the direction of gaze to the bounce point), the gaze moved down (Fig. 4, bottom). The batsmen varied their downward head velocity to create these differences in gaze movement. However, their eyes made slightly upward movements for all balls (except for Mark on over-pitched balls, Fig. 5a). For over-pitched balls, players seemed to begin tracking before the bounce, immediately following the saccade.

\section{Variation between individual batsmen}

Mark, Richard and Charlie's overall visual strategy was similar, despite their widely different skill levels. They watched the delivery of the ball, made saccades to the bounce point before the ball got there, tracked the ball accurately for at least $0.2 \mathrm{~s}$ after the bounce, then more loosely tracked the ball on its final approach to the bat. However, within this common overall strategy, there were differences that seemed to reflect their abilities.

Mark, the best batsman, showed more pursuit tracking than Charlie or Richard, relying on a combination of saccade and pursuit to get to the bounce point (Fig. 4). Thus, for good length balls, the saccade accounted for only $48 \pm 11 \%$ (mean \pm s.d.) of the total pre-bounce gaze change for Mark, compared to $77 \pm 12 \%$ for Charlie and $69 \pm 8 \%$ for Richard. The differences were significant between Mark and Charlie and between Mark and Richard ( $t$-test, $p<0.002$ and $p<0.01$, respectively), but were not significant between Charlie and Richard $(p>0.1)$. On the over-pitched balls, Mark used smooth pursuit almost exclusively (Fig. 5a). (In baseball, professional batters also have good tracking abilities ${ }^{8}$.)

Richard, the batsman with the least skill, was slower to respond to the appearance of the ball, taking at least $0.2 \mathrm{~s}$ to initiate a saccade. The times to the midpoints of his saccades were consistently higher than the other player's times (Fig. 5b). In the range of bounce distances from 7.5 to $10 \mathrm{~m}$, the differences in mean times for Mark and Charlie were not significant ( $t$-test, $p>0.1$ ); however, the differences between theirs and Richard's times were highly significant $(p<0.001)$. The timing of Richard's
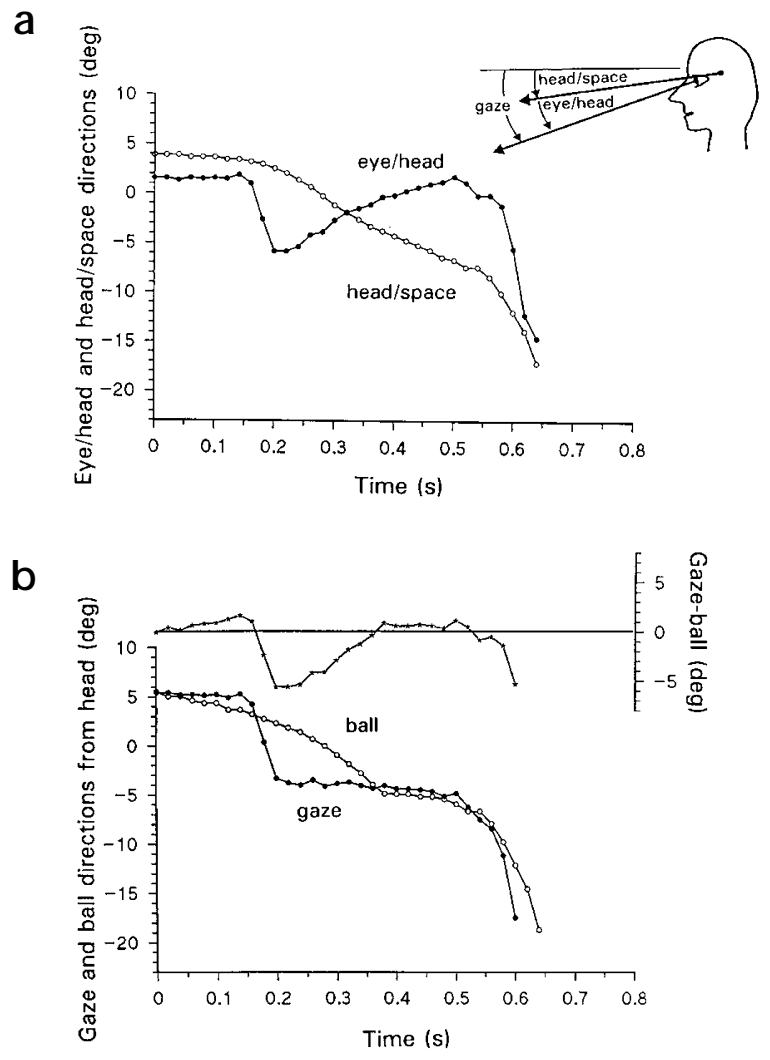

Fig. 3. Relations of visual variables and ball direction during the delivery illustrated in Fig. 2. Direction of the eyes and head (a) and the gaze and ball (b). Descriptions of angles in inset. Upper record on (b) shows gaze error, the difference between ball direction and gaze direction. Error is minimal at the moment of delivery and the bounce point, but is large in between.

saccades were also more variable than the other batsmen's saccade timings (Fig. 5b). Several of Richard's normal speed saccades, occurring at different times, were averaged, which caused an apparently low velocity of his saccades (Fig. 4). Comparing Richard's responses to very short balls with Charlie's or Mark's responses (Fig. 4), it seemed that Richard was not anticipating the movement of the ball, and was waiting until it completed a large part of its flight to the bounce point before starting the saccade. This 'catch-up' saccadic behavior is expected of someone who has not played cricket. With the medium-speed deliveries used here, Richard's viewing technique was adequate because he was (just) in a position to see the bounce on all deliveries. However, with a faster bowler, Richard's response would have been inadequate. If a ball bounced $0.2 \mathrm{~s}$ after delivery, Richard would be too late to see it. Either he would not have started his saccade at this point, or the bounce would have occurred at mid-saccade, during which saccadic suppression would briefly suspend vision. By comparison, even with the very short balls (those that bounce soonest after delivery) Mark and Charlie reached the bounce point 100 ms before the ball (Fig 4). Indeed, Richard could not manage deliveries faster than the ones we used. (Speed had to be restricted for safety reasons.)

In comparing the good (Mark and Charlie) and the poor (Richard) batsmen, we suggest that the main aspect of oculomotor behavior that is related to batting performance is the speed 
Fig. 4. Average gaze and ball trajectories for each player facing very short, short, good length and over-pitched deliveries (short balls bounce far from him, long ones bounce near him). The length of delivery and the batsman's skill level have systematic effects on the pattern of gaze movements. Gaze angles, means from between 5 and 7 deliveries. Standard deviations, every $100 \mathrm{~ms}$. For each set, the mean time at which the ball bounced is marked on the ball trajectories by an arrow.

and variability of the initial saccade. The skill difference between the expert batsman (Mark) and the good batsman (Charlie) is possibly due to Mark's subtle combination of pursuit tracking and saccadic movement as he locates the bounce point. There seemed to be no systematic differences in the way the three batsmen tracked the ball after the bounce.

\section{Discussion}

Batsmen facing fast bowlers do not keep their eye on the ball throughout its flight. They fixate on it as it is delivered, at the time of the bounce and for a period up to about $200 \mathrm{~ms}$ after the bounce. Because Mark and Charlie hit all the balls (and Richard hit most of them), it is evidently not necessary to track the ball between release and bounce nor for more than about $200 \mathrm{~ms}$ after the bounce.

Top batsmen emphasize the need for early information about the trajectory of the ball. "In a perfect world, you will see the ball early and play it late" (Geoffrey Boycott). "The key to playing all strokes is to see quickly the line and length of the ball and to move early into the appropriate position" (David Gower) ${ }^{10}$. In the introduction, we argued that image expansion information (the tau theory ${ }^{11}$ ) was unlikely to be the principal method of determining the time of contact between ball and bat. This is reinforced by the finding that the batsmen are not looking at the
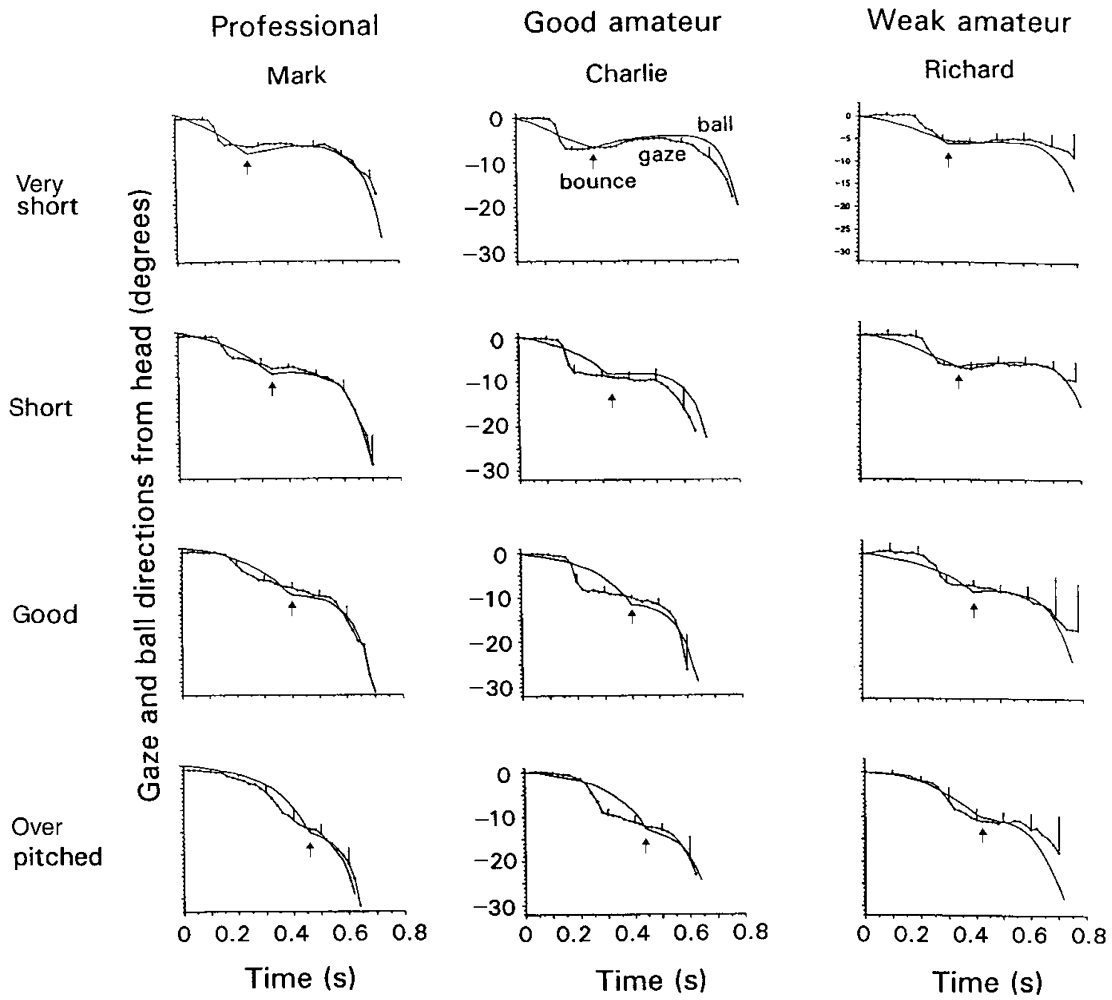

ball for most of the pre-bounce period (Figs. 3 and 4), which is when one would expect they would try to obtain early expansion information. Here we examine the information that is available in the period up to and immediately following the bounce, and we demonstrate that this information is sufficient to allow the batsman to estimate $y$ and $t_{1}$ (Fig. 1).

Batsmen pick up some trajectory information during the first $100-150$ ms of the ball's flight (Fig. 5), as demonstrated by the different latencies of the initial saccade (Mark and Charlie), and by the size of the saccade (Mark). However, this information is ambiguous, because to the batsman, a slow and short delivery will have the same initial downward angular velocity as a fast and long delivery. We suggest that this information is principally used
Fig. 5. The initial downward saccades vary in timing and size depending on the identity of the batsman, and the length of the delivery. (a) Initial eye-in-head saccades, showing different strategies. Each line is the average (over the time axis to avoid distorting the saccades) of the same 5-7 deliveries as in Fig. 4. N ote that latency varies with delivery length for Mark and Charlie. For short and very short balls, Richard's saccades start almost 100 ms later than Mark and Charlie's saccades. VS, very short; S, short; G, good; OP, over pitched. (b) Relationship between the timing of the midpoint of the initial saccade to delivery length $\left(\mathrm{x}_{0}\right)$. For all three batsmen, there is a significant correlation, but it is weakest for Richard (Mark, $r=0.82, p<0.001 ;$ Charlie, $r=0.84, p<0.001$; Richard, $r=0.51, p<0.05)$. Upper abscissa, downward angular velocity of the ball at the batsman's eye before the saccade. a

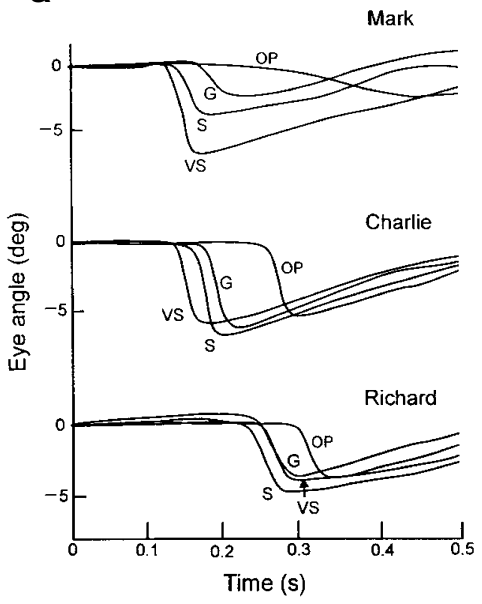

b

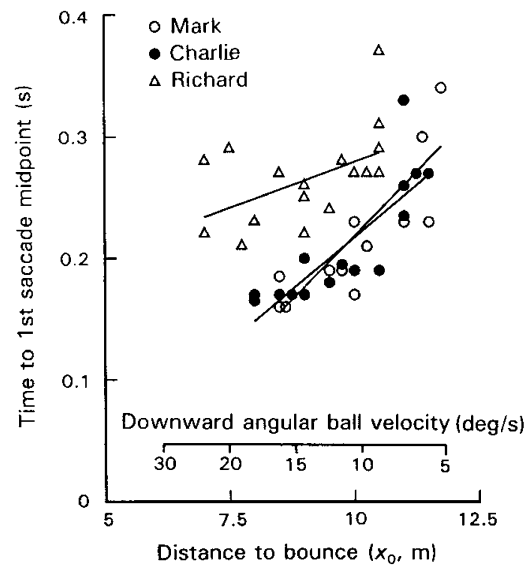


to get gaze direction close to the bounce point, from which unambiguous information is available.

Batsman can make two straightforward measurements, the declination of the bounce point relative to the horizontal ( $\phi$, Fig. 1), and the timing of the bounce relative to the instant of delivery $\left(t_{0}\right)$. The three batsmen always fixated on the delivery and the bounce, the latter being salient because of the discontinuity in the ball's velocity (Figs. 3 and 4); measurement of $\phi$ and $t_{0}$ was consistent with our observations. How are these measurements related to the physical variables that determine $y$ and $t_{1}$ ? Working backward from the contact point, $y$ and $t_{1}$ are uniquely specified by $v_{\mathrm{v} 1}$ and $v_{\mathrm{h} 1}$, the horizontal and vertical velocities of the ball after the bounce, and by $x_{1}$, the distance of the bounce from the batsman. This distance $x_{1}$ is available to the batsman; it is given by $\mathrm{B} / \tan \phi$, where $\mathrm{B}$ is the height of the batsman's eye, which we assume he knows. If the ball were perfectly elastic and the wicket smooth and hard, the postbounce velocities would be the same, apart from a sign change, as the pre-bounce velocities. This, however, is never the case, and $v_{\mathrm{v} 1}$ and $v_{\mathrm{h} 1}$ are related to the pre-bounce velocities via a pair of constants $\left(\mathrm{k}_{\mathrm{v}}\right.$ and $\left.\mathrm{k}_{\mathrm{h}}\right)$, which will vary with the hardness of the pitch. We will return later to how batsmen might obtain these constants.

The pre-bounce velocities are uniquely determined by three variables: the initial velocities of the ball $\left(v_{\mathrm{v} 0}\right.$ and $\left.v_{\mathrm{h} 0}\right)$, and the height from which the ball is delivered $(\mathrm{H})$. Before the bounce, the vertical velocity increases under gravity, and both velocities are slightly decreased by air resistance, but no new variables are involved. The batsman has no direct knowledge of $v_{\mathrm{v} 0}$ and $v_{\mathrm{h} 0 \text {, }}$ but the position and timing of the bounce $\left(x_{0}\right.$ and $t_{0}$, which he can measure) are uniquely related to $v_{\mathrm{v} 0}, v_{\mathrm{h} 0}$ and $\mathrm{H}$. Thus, the pre-bounce ball velocities are mapped onto $x_{0}, t_{0}$ and $\mathrm{H}$. All three of these variables are available. The distance between release point and batsman $(\mathrm{L}=18.5 \mathrm{~m})$ minus $x_{1}(\mathrm{~B} / \tan \phi)$ gives $\mathrm{x}_{0}$. Measurement of the time from delivery to bounce gives $t_{0}$. H can be estimated from the batsman's height B plus $L \tan \theta$, where $\theta$ is the angle of the delivery point from the batsman's eye level (Fig. 1). $\mathrm{H}$ will change little among bowlers.

We have shown that two variables that the batsman can obtain immediately after the bounce ( $\phi$ and $t_{0}$ ) map onto the pre-bounce ball velocities. These convert to post-bounce velocities via two constants $\left(\mathrm{k}_{\mathrm{v}}\right.$ and $\left.\mathrm{k}_{\mathrm{h}}\right)$, which, with $x_{1}$ (again measured from $\phi$ ), determine the time and height of bat-ball contact. A batsman should know the constants (B and L) implicitly. Thus, a mapping exists from $\phi$ and $t_{0}$ onto $y$ and $t_{1}$. To confirm this, we calculated an example of such a mapping (Fig. 6), using the appropriate ballistic equations and making a suitable correction for air resistance (M.F.L. \& P.M., unpublished data). We chose plausible values for $\mathrm{k}_{\mathrm{v}}$ and $\mathrm{k}_{\mathrm{h}}(0.7$ and 0.9$)$ and a delivery height $(\mathrm{H}, 2 \mathrm{~m})$. The surfaces change smoothly with no problematic discontinuities (Fig. 6), but they are not linear either. They show roughly what one would expect: short fast deliveries ( $\phi$ and $t_{0}$, both low) bounce high, over-pitched deliveries (high $\phi$ ) bounce low (Fig. 6a). Time-from-bounce $\left(t_{1}\right)$ is linearly proportional to time-to-bounce $\left(t_{0}\right)$ but at a rate that varies with bounce point ( $\phi$, Fig. 6b).

We are not suggesting that batsmen calculate these mappings each time they face a bowler; rather we assume that they have acquired the mappings after years of practice. There seems to be no conceptual problem in suggesting that a batsman's brain can handle a double mapping of this kind. In driving, for example, steering and speed are simultaneously adjusted to the complex changing visual geometry of the road ahead, and in many sports, actions are based on more than one variable.

What happens when a batsman goes out to play on a wicket
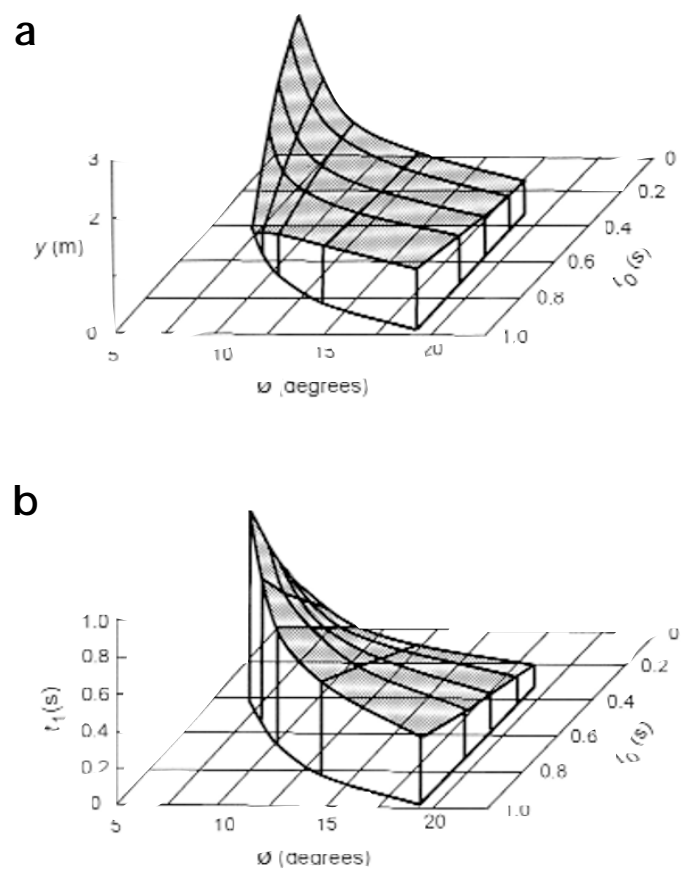

Fig. 6. To hit a ball at the right place and time, based on information obtained at the time of the bounce, a batsman would need to know the two sets of relationships shown by these surfaces. (a) Relationship between the height of the ball at the bat $(y)$ to the two variables the batsman can measure: the declination of gaze at the bounce point $(\phi)$ and the time from leaving the bowler's hand to the bounce $\left(t_{0}\right)$. (b) Relationship between the time the ball takes to reach the batsman from the bounce point $\left(t_{1}\right)$ and the same two variables $\left(\phi\right.$ and $\left.t_{0}\right)$. All plots assume $B=1.5 \mathrm{~m}, \mathrm{H}=2 \mathrm{~m}, \mathrm{~L}=18.4 \mathrm{~m}, \mathrm{k}_{\mathrm{h}}=0.9$ and $\mathrm{k}_{\mathrm{v}}=0.7$.

of unknown hardness (that is, unknown $\mathrm{k}_{\mathrm{v}}$ and $\mathrm{k}_{\mathrm{h}}$ )? Typically, batsmen play defensive shots for a number of deliveries while they 'get their eye in'; these shots do not require accurate judgment of the time or height at which the ball will reach the bat. Then, they attempt shots that require accurate estimates of $y$ and $t_{1}$. We suggest that they use these first few balls to make appropriate adjustments to the mappings (Fig. 6). Computationally, this means changing the values of $\mathrm{k}_{\mathrm{v}}$ and $\mathrm{k}_{\mathrm{h}}$. Practically, it means that a softer wicket will lower the values of $y$ (Fig. 6a) and raise those for $t_{1}$ (Fig. 6b). For a first approximation, these modifications simply involve multiplying all the points on each surface by an appropriate constant. Getting these adjustments exactly right is likely to be a high-order skill, as shown by the amount of practice that even top-class batsmen need when playing in a country with a different climate, and hence on wickets with different properties.

What happens in the period after the bounce? All three batsmen tracked the ball for up to $200 \mathrm{~ms}$, and on the scheme we have proposed, this would seem to be unnecessary. The adjustments just described require an evaluation of the post-bounce behavior of the ball, which could be obtained by observing the rate of change of $\phi$ after the bounce, and also by attemping to see where and when it contacted the bat. Also, the ball can move laterally in unpredictable ways when it bounces, especially if it makes contact on the seam. Batsmen need to watch for this and switch to a defensive stroke if necessary. Finally, we do not rule out the possibility that the 'late' cues of image expansion and change of binocular disparity may become available at this stage to refine the 
estimates of time and position of contact already obtained.

We have shown that it is possible to obtain the information for an attacking shot in cricket from the early part of the ball's flight, including the bounce, and our eye-movement data support the proposal that batsmen use this information. The data do not disprove the involvement of image expansion, changes in disparity or other aspects of the ball's trajectory, however. These other factors must be involved when facing a slow bowler (where it is essential to see what happens to the ball after it has bounced), when facing a ball that reaches the batsman without bouncing (a full toss, relatively rare in cricket), and when batting in baseball (where the ball does not bounce). However, when batting against a fast bowler, early infomation is crucial, and our proposal fits the task well.

\section{Methods}

Batsmen and batting conditions. The three batsmen were Mark, a professional cricketer who has opened the batting for Warwickshire, Charlie, a successful amateur who plays Minor Counties cricket for Oxfordshire, and Richard, an enthusiastic but incompetent amateur who plays low-level club cricket. There was a clear order of ability: Mark $>$ Charlie $>>$ Richard.

The recordings were made in the indoor practice nets at the University of Oxford cricket school. Besides wearing the eye camera on their heads $(235 \mathrm{~g})$, a backpack containing the video recorder $(4.5 \mathrm{~kg})$, and normal batsmen's protective clothing, they were unencumbered. Balls were delivered from a Bola (Cotham, Bristol, UK) bowling machine $18.5 \mathrm{~m}$ from the batsman at a speed of about $25 \mathrm{~m} / \mathrm{s}$ ('medium pace'). The angle at which the ball was delivered was varied, so that the bounce occurred between 0.25 and $0.5 \mathrm{~s}$ after the leaving the machine, equivalent to distances in the range of 12 to $3 \mathrm{~m}$ from the batsman. The ball reached the batsman after approximately $0.75 \mathrm{~s}$ (Fig. 4). (The ball slows down after bouncing, so balls that bounce near the batsman take less time to reach him than balls that land far from him.) Balls were delivered in sequences of 36 or 48 , with the bounce point varied at random from ball to ball. The batsmen were encouraged to play naturally, making whatever shot, defensive or attacking, seemed appropriate to each ball. Mark and Charlie pulled the short deliveries (an attacking shot), drove the over pitched ones (another attacking shot), and played defensively to the good length balls. Richard drove the over-pitched balls and played defensively to the remainder. The pull requires more accurate $\left(y, t_{1}\right)$ judgment than the drive. Richard's eye movement strategy probably gave him insufficient information to play a pull shot.

Eye movement recording. Eye movements were recorded with a device previously used to study the eye movements of drivers ${ }^{12,13}$ and pianists ${ }^{14}$. A single head-mounted video camera recorded the scene ahead and an image of the left eye as it moved in its orbit. The direction of view relative to the head was determined from the position of the iris's outline; a dot corresponding to foveal direction was added to the display of the scene ahead. The accuracy of gaze direction measurement on the scene is about $1^{\circ}$ (roughly the width of the spot on Fig. 2). The sampling rate was $50 \mathrm{~Hz}$. When the batsman's head moved, the field seen by the camera moved within the frame, and by tracking the coordinates of a distant object in the field, the magnitude and direction of head movements was obtained. In a similar way, the position of the ball in the batsman's field of view could also be followed.

Glossary of cricketing terms. Cricket bowlers fall into a number of different classes based on their speed. Fast bowlers (speeds up to $40 \mathrm{~m} / \mathrm{s}$ ) try to beat the batsman by sheer pace, getting the ball past him before he has time to play a shot. Medium pace bowlers (around $25 \mathrm{~m} / \mathrm{s}$ ) aim to beat the bat by making the ball swing laterally in the air ${ }^{1,15}$. Slow bowlers (around $15 \mathrm{~m} / \mathrm{s}$ ) rely on spinning the ball so that it changes direction unpredictably after hitting the wicket.

Length refers to the distance that the ball lands from the batsman. A short ball lands far from him; an over-pitched ball lands close to him. Between these two lengths is a 'good' length, the place that allows the ball time to deviate unpredictably after bouncing, but does not allow the batsman time to adjust his shot ${ }^{3}$. Although a bowler aims to bowl a good length most of the time, for tactical reasons he bowls a variety of lengths. The batsman usually tries to play an attacking shot to short or overpitched balls (an entirely different shot in each case) and a defensive shot to a good length ball. Quick and accurate judgment of length is an essential of successful batting, because it underlies appropriate shot selection.

The wicket is the strip of grass on which the game is played. The condition of the ground influences the way the ball bounces, and the hardness of the ball changes during the course of a game. When the ball is new, it is hard, and it bounces more than when it is old and soft. A cricket ball has two smooth leather faces joined by a stitched seam. If the ball lands on the seam, it is likely to bounce more than if it lands on the smooth face.

The batsman has the option of playing a purely defensive shot with no obligation to run after hitting the ball. The option for the batsman to play entirely defensively, if he wishes, contributes much to the character of the game (and to its inscrutability as a spectacle to viewers more familiar with baseball).

\section{ACKNOWLEDGEMENTS}

We thank J. Horwood for several months of video analysis, and to Z. Dienes for commenting on the manuscript. Support came in part from a grant from the Gatsby foundation.

\section{ReCEIVED 31 MAY; ACCEPTED 28 SEPTEMBER 2000}

1. Regan, D. Visual judgements and misjudgements in cricket, and the art of flight. Perception 21, 91-115 (1992).

2. McLeod, P. \& Jenkins, S. Timing accuracy and decision time in high-speed ball games. Int. J. Sport Psychol. 22, 279-295 (1991).

3. McLeod, P. Visual reaction time and high-speed ball games. Perception 16, 49-59 (1987).

4. Bootsma, R. J. \& van Wieringen P. C. W. Timing an attacking forehand drive in table tennis. J. Exp. Psychol. Hum. Percept. Perform.16, 21-29 (1990).

5. Regan, D. \& Gray, R. Visually guided collision avoidance and collision achievement. Trends Cogn. Sci. 4, 99-107 (2000).

6. Hallett, P. E. in Limits of Vision (Vision and Visual Dysfunction) Vol. 5 (eds. Kulikowski, J. J., Walsh, V. \& Murray, I. J.) 44-80 (Macmillan, Basingstoke, UK, 1991).

7. Ripoll, H., Fleurance, P. \& Cazeneuve, D in Eye Movements: From Physiology to Cognition (eds. O’Regan, J. K. \& Lévi-Schoen, A.) 616-617 (Elsevier, Amsterdam, 1987)

8. Land, M. F. \& Furneaux, S. The knowledge base of the oculomotor system. Phil. Trans. R. Soc. Lond. B 352, 1231-1239 (1997).

9. Bahill, A. T. \& LaRitz, T. Why can't batters keep their eyes on the ball. Am. Sci. 72, 249-253 (1984).

10. Lewes T. MCC Masterclass: the New MCC Coaching Book (Weidenfeld \& Nicholson, London, 1994).

11. Lee, D. N., Young, D. S., Reddish, P. E., Lough, S. \& Clayton, T. Visual timing in hitting an accelerating ball. Q. J. Exp. Psychol. A 35, 333-346 (1983).

12. Land, M. F. Eye-head coordination during driving. IEEE Proc. SMC Conf. Le Touquet 3, 490-494 (1983).

13. Land, M. F. \& Lee, D. Where we look when we steer. Nature 369, 742-744 (1994).

14. Furneaux, S. \& Land, M. F. The effects of skill on the eye-hand span during musical sight reading. Proc. R. Soc. Lond. B 266, 2435-2440 (1999).

15. Adair, R. K. The Physics of Baseball (Harper \& Row, New York, 1990). 